\title{
Screening for balance disorders in mildly affected multiple sclerosis patients
}

\author{
Malou H. J. Fanchamps • Henrik Gensicke • \\ Jens Kuhle $\cdot$ Ludwig Kappos • John H. J. Allum • \\ Özgür Yaldizli
}

Received: 17 October 2011/Accepted: 8 December 2011/Published online: 21 December 2011

(C) Springer-Verlag 2011

\begin{abstract}
Multiple sclerosis (MS) patients often complain about balance problems when Romberg's test and tandem gait are normal. The aim of the study was to determine if measures of trunk sway taken during a battery of stance and gait tasks could be used to detect subclinical balance disorders. We recorded trunk angular sway in the pitch and roll directions from $20 \mathrm{MS}$ patients (EDSS $1.4 \pm 0.5$ ) and 20 age- and gender-matched healthy controls (HCs), during 12 stance and gait tasks. We filmed 22 subjects simultaneously. Two neurologists assessed the videos, deciding whether task performance was pathological. Sway measures were significantly different between patients and HCs in eight out of 12 balance tasks. The most significant differences between MS patients and HCs were pitch angle range standing on one leg with eyes open on a firm surface (mean $3.13^{\circ}$ vs. $2.09^{\circ}, p=0.005$ ), and on a foam support surface (mean $6.24^{\circ}$ vs. $2.96^{\circ}, p=0.006$ ), pitch velocity range walking $8 \mathrm{~m}$ with eyes closed (mean $75.5 \mathrm{vs} .50 .2 \%$, $p<0.001)$ and pitch velocity range walking $3 \mathrm{~m}$ on heels (mean 85.37 vs. $60.9 \%$ s, $p=0.002$ ). Multivariate analysis revealed a model with three tasks which detected balance
\end{abstract}

The authors M. H. J. Fanchamps and H. Gensicke contributed equally to this work.

M. H. J. Fanchamps

Department of Neurology, Radboud University Nijmegen

Medical Centre, Nijmegen, The Netherlands

M. H. J. Fanchamps · J. H. J. Allum

Department of Oto-rhino-laryngology, University Hospital

Basel, Basel, Switzerland

H. Gensicke · J. Kuhle · L. Kappos · Ö. Yaldizli ( $\square)$

Department of Neurology, University Hospital Basel,

Petersgraben 4, 4031 Basel, Switzerland

e-mail: YaldizliO@uhbs.ch disorders in $84 \%$ of the MS patients and $90 \%$ of the HCs correctly. The neurologists achieved accuracies of $30 \%$ for the MS patients and $82 \%$ for the HCs. Using trunk sway measures during stance and gait tasks is a sensitive screening method for balance problems in MS patients, and is more accurate than assessment by trained neurologists.

Keywords Balance - Multiple sclerosis . Screening procedures $\cdot$ Trunk sway $\cdot$ Gait

\section{Introduction}

In multiple sclerosis (MS) patients detection of balance deficits is challenging because MS patients often complain about balance problems when the Romberg test is negative and gait tests are normal [1]. Other clinical tests, for example, the Berg Balance Scale and Tinetti Performance Oriented Mobility Assessment, are also not sensitive enough to detect minimal balance impairment in MS patients [2]. The main problem with balance tests is the subjective character of the assessment. Quantifying balance control may help to capture MS related minimal or subclinical balance impairment. There is no systematic study aimed to determine which stance and gait tasks should be used to detect subclinical balance disorders in MS patients. During the tasks we measured trunk angular sway because it can be used for quantifying balance control during both types of tasks. To compose a MS specific protocol, we selected tasks commonly used in neurological examinations, those used in previous studies, as well as those known from a parallel study [3] to be correlated with disease severity in MS patients. As we wished to establish if our detection technique was more accurate than assessment of the same tasks by certified neurologists, we compared the results of the discriminant model emerging 
from quantitative analysis of the trunk sway measures with the assessment of the neurologists.

\section{Methods}

Protocol

We created a test battery of six stance tasks and six gait tasks. The battery was performed without shoes. Tasks suggested by Gensicke et al. [3] were standing on one leg with eyes open on a firm surface and foam support surface for $20 \mathrm{~s}$ (s1eo, s1eof). The Romberg test was included but with feet together and performed for $30 \mathrm{~s}$ on a foam support surface [4] with eyes open and closed-abbreviated s30ftfeo and s30ftfec. Tandem stance tasks for 30 s eyes open and closed (s30tseo, s30tsec) were chosen because these showed significant differences between MS patients and HCs [4]. Walking eight tandem steps with eyes closed (w8tsec) is part of the Fregly ataxia battery, a widely used clinical test for the assessment of balance [5]. We also included an eyes open tandem gait task (w8tsec) as suggested by Gensicke et al. [3]. The get up and go $3 \mathrm{~m}$ task (gug) was used in several studies [6-8]. Finally, a number of tasks from standard clinical test batteries were used; walking $8 \mathrm{~m}$ was performed with eyes open and eyes closed (w8meo, w8mec), and walking for $3 \mathrm{~m}$ on the heels (w3mheo). Task duration was as listed above for stance tasks or until the task was completed for gait tasks. The recording was stopped when the subject lost balance or needed assistance from a spotter.

\section{Subjects}

Patients were recruited from outpatients of the Department Neurology at the University Hospital Basel in Switzerland. Inclusion criteria were diagnosis of MS according to the McDonald criteria revised in 2005 [9] or clinically isolated syndrome (CIS), age younger than 40 years and an EDSS score $\leq 2$. Sixteen MS patients (four male and 12 female) and four females with CIS participated, with an average age of 31 (range 23-39) years, an average EDSS score of $1.4 \pm 0.5$, and mean disease duration $6.8 \pm 4.7$ years. Twenty ageand gender-matched healthy subjects acted as controls. Participation was voluntary. All patients and HCs gave their written informed consent to participate. The study was approved by the ethical committee of the University of Basel. The demographic data is presented in Table 1.

\section{Measurement devices}

Trunk movements were measured using a SwayStar ${ }^{\mathrm{TM}}$ device (Balance International Innovations $\mathrm{GmbH}$, Switzerland) which contains two digital gyroscopes registering pitch
Table 1 Subject characteristics

\begin{tabular}{llll}
\hline & Patients & Controls & $p$-value \\
\hline$n$ & 20 & 20 & $\mathrm{~ns}$ \\
Females/males & $16 / 4$ & $16 / 4$ & $\mathrm{~ns}$ \\
Age (years, range) & $30.9(23-39)$ & $30.9(23-39)$ & $\mathrm{ns}$ \\
Height (cm) & $169.6 \pm 11.6$ & $170 \pm 7.1$ & $<0.05$ \\
Weight (kg) & $72.5 \pm 13.8$ & $61.9 \pm 7.2$ & $<0.05$ \\
BMI & $25.3 \pm 4.7$ & $21.4 \pm 2.1$ & $<0.05$ \\
EDSS (range) & $1.4 \pm 0.5(0-2)$ & - & \\
Disease duration from & $6.75 \pm 4.67$ & - & \\
$\quad$ FS (years) & & & \\
Disease duration & $4.58 \pm 2.96$ & - & \\
$\quad$ from FD (years) & & & \\
\hline
\end{tabular}

$B M I$ body mass index, $F S$ first symptoms, $F D$ first diagnosis, $E D S S$ Expanded Disability Status Scale

(posterior-anterior) and roll (medial-lateral) angular velocities. The device is worn at the level of the lower back (L3-L5), near the centre of mass. It is attached to a modified motorcycle kidney belt which is strapped around the waist. The sample rate is $100 \mathrm{~Hz}$, and the drift of the gyroscopes is approximately $1 \% \mathrm{~h}$ (less than the earth's rotation). Twentyseconds of measurement therefore results in a drift of maximally $0.006^{\circ}$.

Assessment by certified neurologists

Eleven subjects in each group were filmed while performing the task battery. The videos were shown to two certified neurologists unknown to each other. Their task was to assess the videos not knowing whether the subject was a healthy control or not, and to decide whether task performance was pathological. Finally they were requested to classify each subject as a MS patient or healthy control. The overall group assignment of the neurologist was compared to that of our discriminant procedure (see below) by means of classification accuracies.

Assessment of subjective balance problems

Before the experiment started, the patients were asked to complete a questionnaire: the Dizziness Handicap Index (DHI) [10]. This is a self-report based on 25 questions which quantify the subjective level of disability and handicap in three subscales (emotional, functional and physical). Questions could be answered with no (score 0), sometimes (2), or yes (4), the final score ranged from 0 to 100. The higher the DHI, the more disability was experienced by the patient $[3,11]$. A score of zero on the DHI was taken as no subjectively perceived balance problem. All healthy control subjects had a score of zero, having reported no subjective balance disorder. 
Data processing and statistical analyses

We used the peak to peak roll and pitch, angle and velocity ranges, as primary outcome measures to compare the MS patients with the HCs (see Fig. 1). Roll and pitch angles of the trunk sway data were derived from the recorded angular velocities by trapezoidal integration. We performed MannWhitney $U$ tests between the peak to peak measures because the data were not normally distributed. We assumed that patients have more sway than healthy control subjects, we used only one-tailed outcomes to decide whether there is a significant difference or not. The significant variables were entered into a stepwise discriminant analysis in order to identify the most sensitive test protocol and measures to distinguish between MS patients and healthy controls. The $F$ values of 3.8 for entry into, and 2.7 for removal from, the discriminant analysis were used. All statistical analysis was performed with SPSS 19.0 for Windows. The significance level was set at 0.05 and corrected for multiple comparisons by using Bonferroni.

\section{Results}

Stance tasks

Three of the six stance tasks showed significant difference between two groups (Table 2).

The tasks and variables with the most significant differences were pitch angle range standing on one leg with eyes open on a firm support surface (means 3.13 vs. $2.09^{\circ}, p=0.005$ ) and on a foam support surface
Fig. 1 Trunk angle and angular velocity plots from typical subjects for the task, standing on one leg on a foam support surface with eyes open. In the angular velocity plots pitch angular velocity ( $y$-axis) is plotted against roll velocity ( $x$-axis)
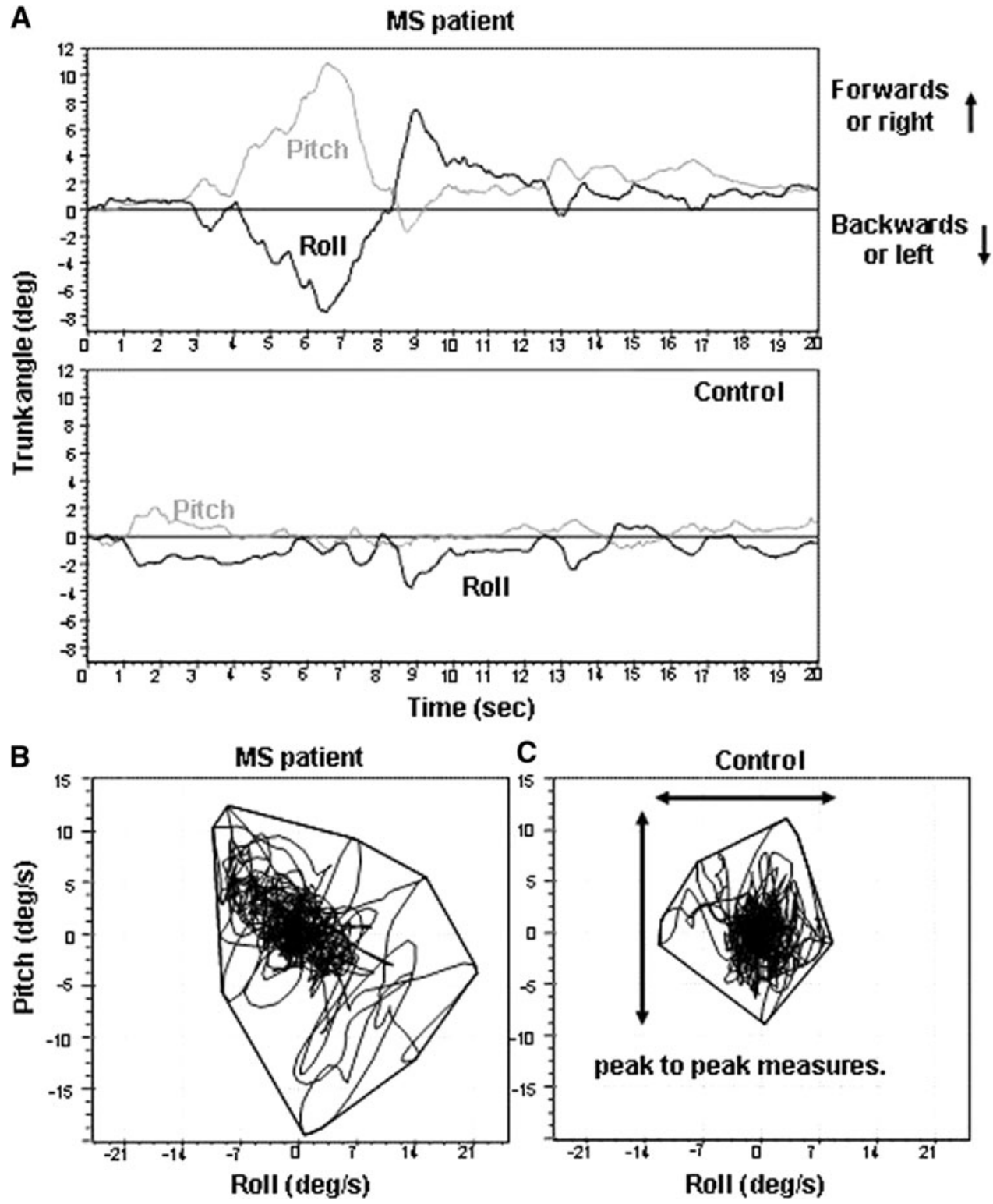
Table 2 Significant trunk sway differences between patients and controls
Results for the eight test protocols with significant differences $(p<0.05)$ with respect to the control group are marked in bold text and by an asterisk $(*)$

sleo standing on one leg eyes open, s30ftfec standing for $30 \mathrm{~s}$ feet together on foam support surface with eyes closed, sleof standing on one leg with eyes open on foam support surface, w8tsec tandem walk for $8 \mathrm{~s}$ with eyes open, gug get up and go $3 \mathrm{~m}$ task, w8meo walking $8 \mathrm{~m}$ with eyes open, w8mec walking $8 \mathrm{~m}$ with eyes closed, w3mheo walking for $3 \mathrm{~m}$ on the heels

\begin{tabular}{|c|c|c|c|c|}
\hline \multirow[t]{2}{*}{ Protocol } & \multicolumn{2}{|l|}{ Angle range $\left({ }^{\circ}\right)$} & \multicolumn{2}{|c|}{ Velocity range $(\% / s)$} \\
\hline & Patients & Controls & Patients & Controls \\
\hline \multicolumn{5}{|l|}{ sleo } \\
\hline Roll & $2.59(1.59)$ & $1.89(0.74)$ & $9.49(7.24)$ & $6.71(5.45)$ \\
\hline \multicolumn{5}{|l|}{$\mathrm{s} 30 \mathrm{ftfec}$} \\
\hline Roll & $2.93(1.08)$ & $2.61(0.94)$ & $10.81(5.69)$ & $8.62(3.45)$ \\
\hline \multicolumn{4}{|l|}{ s1eof } & $7.98(3.76)$ \\
\hline Roll & $9.20(7.58)^{*}$ & $3.92(4.75)$ & $29.24(23.87)^{*}$ & $14.00(12.19)$ \\
\hline \multicolumn{4}{|l|}{ w8tsec } & $10.94(6.04)$ \\
\hline Roll & $11.35(4.37)$ & $10.42(3.98)$ & $46.71(12.60)$ & $41.46(14.87$ \\
\hline \multicolumn{4}{|l|}{ gug } & $37.51(15.73)$ \\
\hline Roll & $5.02(1.07)^{*}$ & $5.75(1.42)$ & $45.50(15.50)$ & 44.05 (17.14) \\
\hline \multicolumn{5}{|l|}{ w8meo } \\
\hline Roll & $5.86(0.87)$ & $6.29(1.77)$ & $61.83(21.66)$ & $58.19(15.14)$ \\
\hline $\begin{array}{c}\text { Pitch } \\
\text { w8mec }\end{array}$ & \multicolumn{3}{|c|}{ w8mec } & $56.87(13.35)$ \\
\hline Roll & $6.07(1.32)$ & $5.89(1.42)$ & $56.70(20.05)$ & $53.21(12.14)$ \\
\hline Pitch & $9.60(2.00)$ & $9.56(2.40)$ & $75.53(30.94)^{*}$ & $50.23(13.34)$ \\
\hline \multicolumn{5}{|l|}{ w3mheo } \\
\hline Roll & $5.68(1.67)$ & $5.52(1.58)$ & $59.16(19.50)$ & $53.29(9.08)$ \\
\hline Pitch & $8.86(3.06)$ & $7.81(2.30)$ & $85.37(26.47)^{*}$ & 60.94 (13.98) \\
\hline
\end{tabular}

(means 6.24 vs. $2.96^{\circ}, p=0.006$ ). Figure 1 shows examples of the eyes open on foam one-legged stance task. The following three stance tasks s30tseo, s30tsec, s30ftfeo showed no significant group differences $(p>0.05)$ (Fig. 1).

\section{Gait tasks}

In contrast to the stance tasks five of the six gait tasks showed significant differences between MS patients and healthy control subjects (see Table 2). The most significant differences were seen for the pitch velocity range walking $8 \mathrm{~m}$ with eyes closed (means 75.5 vs. $50.2^{\circ} / \mathrm{s}, p<0.001$ ) and in the pitch velocity range walking $3 \mathrm{~m}$ on heels (means 85.37 vs. $60.94 \%$ s, $p=0.002$ ). Table 2 shows that almost all the group differences in gait are for pitch angular velocity ranges and not for angle ranges.

\section{Discriminant analysis}

Figure 2 provides scatter plots for each subject on completion of the discriminant analysis. The classification function calculated was:
$0.36 \times$ Pitch velocity range $($ w8mec $)+0.81$ Roll angle range (s1eof) $-5.99 \times$ Roll angle range $($ gug $)+0.464$ (Fig. 2).

We performed the step-wise discriminant analysis with all variables which were significant different based on the Mann-Whitney $U$ tests (see Table 2). Pitch velocity range recorded during walking $3 \mathrm{~m}$ on heels, eyes open emerged as the best discriminator in step 1, but it was removed in step 4 due to correlations with the other entered variables. The lowest $F$ value from the ANOVA analysis for the variables to enter the discriminant function was 8.5 with 3.35 dof $(p<0.001)$. The approximate final $F$ value for the MANOVA analysis of group means for variables in the discriminant function was 9.5 with 3.35 dof $(p<0.001)$. When differentiating the MS patients from the control group, the overall classification was $87 \%$. For patients the classification accuracies was $84 \%$ and for healthy controls the classification accuracies was $90 \%$. In Table 3 the details of the discriminant analysis classification accuracy are shown and compared to the neurologists classifications (Table 3). 


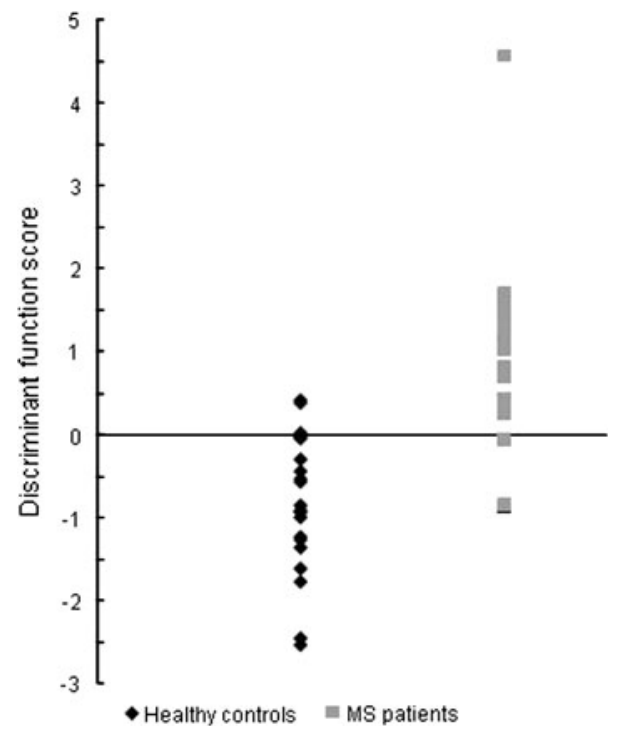

Fig. 2 Scatter plots of patient and healthy control discriminant function values

Table 3 Classification tables for discriminant analysis model and neurologist rating

\begin{tabular}{llll}
\hline Classification by discriminant model & \multicolumn{2}{l}{ Actual status } \\
\cline { 2 - 4 } & Patient & Control & Total \\
\hline $\begin{array}{l}\text { Predicted group for discriminant } \\
\text { model }\end{array}$ & & & \\
Patient & $16(85 \%)$ & 2 & 18 \\
Control & 3 & $18(90 \%)$ & 21 \\
Total & 19 & 20 & 39 \\
Selection by neurologist 1 & & & \\
Predicted group for neurologist 1 & & & \\
$\quad$ Patient & $3(30 \%)$ & 2 & 5 \\
Control & 7 & $9(82 \%)$ & 16 \\
$\quad$ Total & 10 & 11 & 21 \\
Selection by neurologist 2 & & & \\
Predicted group for neurologist 2 & & & \\
$\quad$ Patient & $3(27 \%)$ & 6 & 9 \\
$\quad$ Control & 8 & $5(45 \%)$ & 13 \\
$\quad$ Total & 11 & 11 & 22 \\
\hline
\end{tabular}

Number and percentages of patients and controls with correct and incorrect classifications are listed

Comparison between neurologists' assessments and discriminant analysis

The first neurologist had classification accuracies of $30 \%$ for MS patients and $82 \%$ for controls, an overall score of $57 \%$, and the second neurologist had classification accuracies of $27 \%$ for MS patients and $45 \%$ for controls, an overall score of $36 \%$ (see Table 3). Walking eight tandem steps with eyes closed was mostly seen as pathological in
HCs (11 times), followed by tandem stance with eyes closed (seven times) and standing on one leg on foam with eyes open (five times). The tasks of our discriminant model were assessed six times as pathological in healthy controls by the neurologists.

\section{Subjective balance problems}

Eleven $(55 \%)$ of the 20 patients had a DHI score of $>0$. They answered one or more questions with at least "sometimes" and, based on this criterion, classified themselves subjectively as having a balance problem. Our discriminant model confirmed the presence of balance disorders in nine of these 11 patients. Only two of these patients had a positive Romberg result, the same two patients identified as pathological by the neurologists. Among the patients who stated they had no balance problems in the DHI questionnaire (zero score), one patient who also had a negative Romberg test was classified as a control with both techniques neurologist's selection and our discriminant analysis.

\section{Discussion}

The main outcome of our study was that among 12 clinical tests, standing on one leg eyes open on a foam support surface, walking $8 \mathrm{~m}$ with eyes closed and get up from a stool and go $3 \mathrm{~m}$ emerged as the best clinical test battery to screen for subclinical balance disorders in MS patients. This mix of a stance, gait and the get up and go task covers different aspects of balance control. This test battery is able to detect subclinical balance problems in MS patients more accurately than the standard neurological examination using the Romberg test, normal walking with eyes open, and the tandem gait test with eyes open. In this study the sway measures of healthy subjects without any balance problems served as the comparison ("gold") standard.

It is an open question what should serve as the standard for comparison. If we had taken the patients subjective assessments based on the DHI scores higher than zero, then our model identified $82 \%$ of these patients correctly, a similar figure to the $84 \%$ correct identification across all patients. This is a question that needs to be explored in future studies. Two neurologists, independent from each other and blinded to the clinical data of the patients, were requested to attempt to select the patients on the basis of presumed balance deficits by watching videos of the patients and healthy controls performing the 12 balance tasks of the study. However, they only achieved accuracies of $27-30 \%$ in detecting balance deficits, whereas assessment of sway measures in the three tests mentioned above could detect balance disorders in $84 \%$ of the patients. 
Currently, the Romberg test is mostly used clinically to identify an abnormal balance control in neurological patients. Beside the Romberg test, there are more clinically based tests, e.g. Berg Balance Scale and Tinetti Performance Oriented Mobility Assessment. These tests are partly based on subjective ratings and provide no guarantee that there is no balance problem when the assessment is negative. There are MS patients who complain about subjective discomfort of balance even if there is no clinical evidence of any balance problem [1]. Detecting minimal impairment of balance based on observation is not easy as confirmed by our neurologists who examined the videos. Perhaps their task would have been easier if they were informed, based on the results of our discriminant analysis, to concentrate on excessive trunk roll angle for the onelegged stance task on foam, increased trunk pitch velocities during walking with eyes closed, and reduced trunk roll angles for the get up and go task. Presumably, for them to judge the greater trunk pitch velocity in MS patients during walking would be difficult. In this respect, the question also arises whether the pitch velocity during walking on the heels might be easier to employ than reduced roll during the get up and go test. The former measure replaced the latter in the step-wise discriminant analysis.

The test battery we started with contained six different stances and six gait tasks; in eight of these tasks we detected a difference in trunk sway between MS patients and healthy controls. Five of these were gait tasks. This corresponds to findings of Karst et al. [2], who concluded, based on literature that balance control during stance is less discriminating than during gait. Our analysis revealed that standing on one leg on foam with eyes open showed the greatest difference in trunk sway between MS patients and healthy controls, thus confirming the findings in Gensicke et al. [3]. In MS, proprioceptive deficits delay balance corrections [12]. Thus, it was not surprising that many tests showing significant differences relied on further reducing the effectiveness of proprioceptive inputs using a foam support or placing greater reliance on this input by performing the tests eyes closed. Thus, it appears easier to detect the minor subclinical balance problems during stance when the effect of the problem, a greater trunk sway, is enhanced by decreasing the proprioceptive input or removing the possibility for visual compensation of weakened proprioceptive inputs.

We assumed that MS patients would have more trunk sway when performing the stance and gait tasks. In Table 2, where the differences are presented, there is only one task where patients had less trunk sway comparing to healthy subjects. A significant lower trunk sway was seen for the roll angle range in the get up and go $3 \mathrm{~m}$ task. This is also one of the discriminators in our screening model. It should be noted that this is the task with the greatest sway angles. Karst et al. [2] found in their study, on voluntary leaning and reaching, a smaller displacement of the centre of pressure in minimally impaired MS patients compared to healthy controls. They suggested that the MS patients were instinctively aware of their limitations and found a strategy to achieve the same movements, but with less displacement of the centre of pressure, and thus with less trunk sway. Martin et al. [13] found also a worse performance (smaller reaching movements) of MS patients in voluntary reaching compared to HCs. It is possible that MS patients attempt to keep their lateral instability to a minimum during the get up phase.

The discriminant model had a quite reasonable overall classification accuracy of $87 \%$. It was better than the neurologists, who had overall accuracies of 57 and 36\%. Our neurologists classified 82 and $45 \%$ of the controls correctly compared to our model with $90 \%$. However, the classification of 30 and $27 \%$ for the patients is much worse then the $84 \%$ of our discriminant model. The neurologists marked different tasks as pathological compared to our discriminant model, except for s1feo. The tasks s30tsec and w8tsec were more often marked as pathological for incorrectly classified healthy subjects. It appears that the range of normal trunk sway for these tasks is greater than the neurologists estimated. With the three task battery of tests we developed using trunk sway measures of balance control; we have an objective measurement to detect balance disorder in minimally impaired MS patients. In the patient group more patients were recognized as such. Not only is the better classification an advantage from the point of screening for MS, but it is also an objective measurement which could be used to monitor the effect of medication.

In our patient group, 11 of the 20 patients classified themselves subjectively as having a balance problem. Only two patients were classified as a patient by the neurologists, the same two with positive Romberg results. However, nine of these 11 including the two with positive Romberg results were classified as having a balance problem with our discriminant technique. This confirms two previous findings. Firstly that the Romberg test is not sensitive in detecting subclinical balance disorders [1, 2], and secondly that clinical balance tests do not identify the balance problems of MS patients with subjective balance impairment [14].

A number of factors may have influenced our comparisons between MS patients and controls. Our classification percentages of MS patients can be viewed as sensitivity and specificity measures as all patients had MS according to actual diagnosis criteria [9]. Matched controls did not have an MRI. Thus, we can not exclude the possibility that the controls had lesions in the CNS affecting balance system subclinically. However, the prevalence of subclinical MS lesions in the brain of 20 healthy subjects can be estimated as insignificant small [15]. An overall computation of sensitivity of our discriminant technique would require MRIs for the controls. Our healthy controls are 
age- and gender-matched with the MS patients (see Table 2). Height has no effect on trunk sway over the age range of the patients [16], but the distribution of weight may influence the stability, the trunk sway, and thus the balance of a person. We did not match the healthy controls according to weight, but to age and gender. The healthy controls had, on average, a significant lower BMI, based on a significant lower weight. Some patients could not maintain their balance during the whole task. We only included data taken when subjects were maintaining their balance. Some of the stance tasks had, therefore, shorter durations than those of normal subjects. One patient could not do several tasks, including the discriminating tasks entered into the discriminant model. Therefore, we only had 19 patients in the discriminant analysis who were classified. Although we have patients with a low EDSS and relative short disease duration, some are taking MS related medication which may have effect on their balance. In total 14 patients were on medication (natalizumab seven, interferon beta $1 \mathrm{~b}$ five, and others two). However, in clinical phase III studies vertigo and/or balance problems were not reported to be significantly higher in the treatment group compared with placebo [17-22].

In conclusion, using trunk sway measures during stance and gait tasks provides a sensitive means of screening for balance problems in mildly affected MS patients, and is more accurate than the current practice, assessment by a certified neurologist. However, further work will need to examine whether this technique can be used for follow-up of patients and assessment of potentially effective symptomatic physical or drug therapy.

Acknowledgments MHJ Fanchamps was supported by a StudEx grant and Prof. JHJ Allum was supported by the National Swiss Research Foundation, grant No 32/117950.

Conflicts of interest $\mathrm{H}$ Gensicke, L Kappos, J Kuhle and O Yaldizli have no conflicts of interests regarding this study. JHJ Allum worked as a consultant for the company supplying the equipment (SwayStar ${ }^{\text {TM }}$ ) used in this study.

\section{References}

1. Corradini ML, Fioretti S, Leo T, Piperno R (1997) Early recognition of postural disorders in multiple sclerosis through movement analysis: a modeling study. IEEE Trans Biomed Eng 44:1029-1038

2. Karst GM, Venema DM, Roehrs TG, Tyler AE (2005) Center of pressure measures during standing tasks in minimally impaired persons with multiple sclerosis. J Neurol Phys Ther 29:170-180

3. Gensicke H, Corporaal S, Kappos L, Allum J, Yaldizli Ö (2011) Correlations of balance instability and disability in multiple sclerosis patients [abstract]. J Neurol 258:P92

4. Soyuer F, Mirza M, Erkorkmaz U (2006) Balance performance in three forms of multiple sclerosis. Neurol Res 28:555-562
5. Cattaneo D, Cardini R (2001) Computerized system to improve voluntary control of balance in neurological patients. Cyberpsychol Behav 4:687-694

6. Cakt BD, Nacir B, Genc H, Saracoglu M, Karagoz A, Erdem HR et al (2010) Cycling progressive resistance training for people with multiple sclerosis: a randomized controlled study. Am J Phys Med Rehabil 89:446-457

7. Missaoui B, Thoumie P (2009) How far do patients with sensory ataxia benefit from so-called "proprioceptive rehabilitation"? Neurophysiol Clin 39:229-233

8. Widener GL, Allen DD, Gibson-Horn C (2009) Randomized clinical trial of balance-based torso weighting for improving upright mobility in people with multiple sclerosis. Neurorehabil Neural Repair 23:784-791

9. Polman CH, Reingold SC, Edan G, Filippi M, Hartung HP, Kappos L et al (2005) Diagnostic criteria for multiple sclerosis: 2005 revisions to the "McDonald Criteria". Ann Neurol 58:840-846

10. Jacobson GP, Newman CW (1990) The development of the dizziness handicap inventory. Arch Otolaryngol Head Neck Surg 116:424-427

11. Cattaneo D, Jonsdottir J, Zocchi M, Regola A (2007) Effects of balance exercises on people with multiple sclerosis: a pilot study. Clin Rehabil 21:771-781

12. Cameron MH, Horak FB, Herndon RR, Bourdette D (2008) Imbalance in multiple sclerosis: a result of slowed spinal somatosensory conduction. Somatosens Mot Res 25:113-122

13. Martin CL, Phillips BA, Kilpatrick TJ, Butzkueven H, Tubridy N, McDonald E et al (2006) Gait and balance impairment in early multiple sclerosis in the absence of clinical disability. Mult Scler 12:620-628

14. Findling O, Sellner J, Meier N, Allum JH, Vibert D, Lienert C et al (2011) Trunk sway in mildly disabled multiple sclerosis patients with and without balance impairment. Exp Brain Res 213:363-370

15. Siva A, Saip S, Altintas A, Jacob A, Keegan BM, Kantarci OH (2009) Multiple sclerosis risk in radiologically uncovered asymptomatic possible inflammatory-demyelinating disease. Mult Scler 15:918-927

16. Hegeman J, Shapkova EY, Honegger F, Allum JH (2007) Effect of age and height on trunk sway during stance and gait. J Vestib Res 17:75-87

17. (1998) Randomised double-blind placebo-controlled study of interferon beta-1a in relapsing/remitting multiple sclerosis. PRISMS (Prevention of Relapses and Disability by Interferon beta-1a Subcutaneously in Multiple Sclerosis) Study Group. Lancet 352:1498-504

18. IFNB Multiple Scleriosis Study Group (2001) Interferon beta-lb is effective in relapsing-remitting multiple sclerosis. I. Clinical results of a multicenter, randomized, double-blind, placebo-controlled trial. 1993 [classical article]. Neurology 57:S3-S9

19. Jacobs LD, Cookfair DL, Rudick RA, Herndon RM, Richert JR, Salazar AM et al (1996) Intramuscular interferon beta-1a for disease progression in relapsing multiple sclerosis. The multiple sclerosis collaborative research group (MSCRG). Ann Neurol 39:285-294

20. Johnson KP, Brooks BR, Cohen JA, Ford CC, Goldstein J, Lisak RP et al (1995) Copolymer 1 reduces relapse rate and improves disability in relapsing-remitting multiple sclerosis: results of a phase III multicenter, double-blind placebo-controlled trial. The copolymer 1 multiple sclerosis study group. Neurology 45:1268-1276

21. Polman CH, O'Connor PW, Havrdova E, Hutchinson M, Kappos L, Miller DH et al (2006) A randomized, placebo-controlled trial of natalizumab for relapsing multiple sclerosis. N Engl J Med 354:899-910

22. Brandes DW, Shaya FT, Pill MW (2010) Quantifying the role of natalizumab in health and economic outcomes in multiple sclerosis. Am J Manag Care 16:S171-S177 